\title{
Design of Flight Control System Using Gain Schedule Fractional PID Controller
}

\author{
M. Fawzy*, H. T. Dorrah ${ }^{\dagger}$ and O. Abd Elrahman*
}

\begin{abstract}
The goal of this paper is to control the trajectory of the flight path of six degree of freedom flying body model using Fractional PID controller (FPID) and Gain Schedule Fractional PID controller. FPID and gain schedule FPID controllers gains with non linear flying body simulation are tuned by Simulink design optimization. Gain Schedule FPID controller is able to compensate for constraints that represent physical limits of actuators in pitch angle. The gain schedule FPID for the six degree of freedom flying body is designed in two phases. The first phase is boost phase where the thrust force is maximized. The second phase is sustain phase where the thrust force is minimized. The results of gain schedule FPID controller are compared with the results of FPID controller.
\end{abstract}

Keywords: six degree of freedom missile model, Fractional PID controller (FPID), Gain Schedule Fractional PID controller, Simulink design optimization.

\section{Nomenclature}

$\begin{array}{ll}C_{x}, C_{y}, C_{z} & \text { Aerodynamic force coefficients } \\ D & \text { Diameter of maximum cross section area [m] } \\ e & \text { Control error }(\mathrm{e}=\mathrm{r}-\mathrm{y}) \\ F_{x}, F_{y}, F_{z} & \text { Components of total forces acting on missile [N] } \\ G & \text { Gravity force [N] } \\ G_{x}, G_{y}, G_{z} & \text { Gravity force components [N] } \\ g & \text { Gravity acceleration [m/sec2] } \\ I_{x}, I_{y}, I_{z} & \text { Moment of inertia components [[kg.m2/sec] } \\ k_{p}, k_{i}, k_{d} & \text { PID gains } \\ M_{T H x}, M_{T H y}, M_{T H z} & \text { Thrust moment components [N.m] } \\ M_{A x}, M_{A y}, M_{A z} & \text { Aerodynamic moment components [N.m] } \\ M_{x}, M_{y}, M_{z} & \text { Components of total moments acting on missile [N.m] } \\ M & \text { The mass of missile [kg] } \\ m_{x 0}, m_{y \beta}, m_{y 0}, m_{z \alpha}, m_{z 0} & \text { Aerodynamic moment coefficients } \\ R_{x}, R_{y}, R_{z} & \text { Aerodynamic force components [N] }\end{array}$

\footnotetext{
Egyptian Armed Forces, Egypt; fawzy2471972@ gmail.com .

$\dagger$ Cairo University, Faculty of Engineering, Electric Power and Machines Department, Giza, Egypt, dorrahht@aol.com .

$\ddagger$ Egyptian Armed Forces, Egypt; osamos54@ gmail.com .
} 


$r$
$S$
$T$
$T_{x}, T_{y}, T_{z}$
$V_{m}$
$V_{x}, V_{y}, V_{z}$
$X$
$X_{g}, Y_{g}, Z_{g}$
$X_{b}, Y_{b}, Z_{b}$
$X_{V}, Y_{V}, Z_{V}$
$X_{c g}$
$Y$
$Z$
$\Phi, \Psi, Y$
$\alpha, \beta$
$\delta$
$\delta_{\alpha}$
$\delta_{\beta}$
$\rho$
$\lambda$
$\omega_{x}, \omega_{y}, \omega_{z}$
$\omega_{c}$

$T$

$V_{m}$

$V_{x}, V_{y}, V_{z}$

$X_{g}, Y_{g}, Z_{g}$

$X_{V}, Y_{V}, Z_{V}$

$X_{c g}$

$\Phi, \Psi, \Upsilon$

$\alpha, \beta$

$\delta$

$\delta_{\alpha}$

$\delta_{\beta}$
Reference signal

Reference area [m2]

Thrust force [N]

Thrust force components [N]

Missile velocity [m/sec]

Missile velocity components [m/sec]

Range of missile [m]

Ground coordinate

Body coordinate

Velocity coordinate

distance between c.g and the nozzle [m]

Vertical displacement of missile (altitude of missile) [m]

Horizontal displacement of missile [m]

Euler's angles [degree]

Angles of attack [degree]

Fractional derivative

Jet deflection angle in the pitch plane [degree]

Jet deflection angle in the yaw plane [degree]

Air density $[\mathrm{kg} / \mathrm{m} 3]$

Fractional integration

Angular velocity components $[\mathrm{rad} / \mathrm{sec}]$

Critical frequency $[\mathrm{rad} / \mathrm{sec}]$

\section{Introduction and Literature Review}

In recent years, the needs for the quality of automatic control increased due to increased complication of plants and sharper specifications of product. The design of optimal variable structure controllers applied to a six degree of freedom missile model will be addressed in this paper. The six degree of freedom missile model is the solution to get a detailed correct data about the missile trajectory. The paper aims are (1) To evolve a complicated mathematical model of flight trajectory simulation for a hypothetical missile, which can be utilized as a base line algorithm participating for design, analysis, and growth of such a system and perform this model using Simulink to ease the design of its control system (2) evolving control system, by utilizing Gain Schedule Fractional PID control methods, [1, 2].

According to MacKenzie, guidance is defined as the procedure for guiding the rout of an object toward a given point, that in general is moving, [1, 3]. Moreover, the father of inertial navigation, Charles Stark Draper, states that "Guidance depends on main principles and involves devices that are same for vehicles moving on land, on water, under water, in air, beyond the atmosphere within the gravitational domain of earth and in space outside this domain, $[1,4]$. The most rich and ripe literature on guidance is found within the guided missile society. A guided missile is known as a space-navigating unmanned vehicle that carries within itself the means for controlling its flight route, $[1,5]$. Guided missiles have been operational since World War II, $[1,3]$. Today, missile guidance theory includes a broad spectrum of guidance laws as traditional guidance laws, optimal guidance laws, guidance laws depend on fuzzy logic and neural network theory, differential geometric guidance laws and guidance laws based on differential game theory. Very interesting personal accounts of the guided missile evolution through and after World War II can be found in the literature, [1, 6-8]. Moreover, Locke and Westrum put the evolution of guided missile methods into a larger perspective, 
$[1,9,10]$. This paper is organized as follows. Section 2 represents mathematical model of six degree of freedom missile equations and. Section 3 displays Gain Schedule Fractional PID controller design. Section 4 presents control applications and results. Finally, conclusions are discussed in section 5 .

\section{Mathematical Model of Missile}

The model represents the six degree of freedom (6-DOF) equations that break down into those depicting kinematics, dynamics (thrust, aerodynamics, and gravity), command guidance generation systems, and autopilot (electronics, instruments and actuators). The input to this model is start conditions, target motion, and target trajectory description, while the outputs are the missile flight information (range, speed, acceleration, etc.) through engagement.

The essential frames required for subsequent analytical evolutions are the body, ground and velocity coordinate systems. The origins of these coordinate systems are the center of gravity (c.g) for missile. In the ground coordinate system, the $X_{g}-Z_{g}$ lie in the horizontal plane and the $Y_{g}$ axis completes a standard right-handed system and goes up vertically. In the body coordinate system, the positive $X_{b}$ axis corresponds with the missile's center line and it is representing as roll-axis. The positive $Z_{b}$ axis is to the right of the $X_{b}$ axis in the horizontal plane and it is representing as the pitch axis. The positive $Y_{b}$ axis goes up and it is representing as the yaw axis. The body axis system is constant with respect to the missile and moves with the missile. In the velocity coordinate system, $X_{V}$ corresponds with direction of missile velocity $\left(V_{m}\right)$, which linked to the directions of missile flight. The axis $Z_{V}$ completes a standard right-handed system,[1, 11-14].

The pitch plane is $X-Y$ plane, the yaw plane is $X-Z$ plane, and the roll plane is $Y-Z$ plane. The ground coordinate system and body coordinate system are linked to each other through Euler's angles $(\Phi, \Psi, Y)$. The ground coordinate system and velocity coordinate system are linked to each other through the angles $(\theta, \sigma)$. In addition, the velocity coordinate system is linked to the body frame through the angle of attack $(\alpha)$ in the pitch plane and sideslip angle $(\beta)$ in the yaw plane. The angles between various coordinate systems are represented in Fig. 1

$[1,11,12,14,15]$.

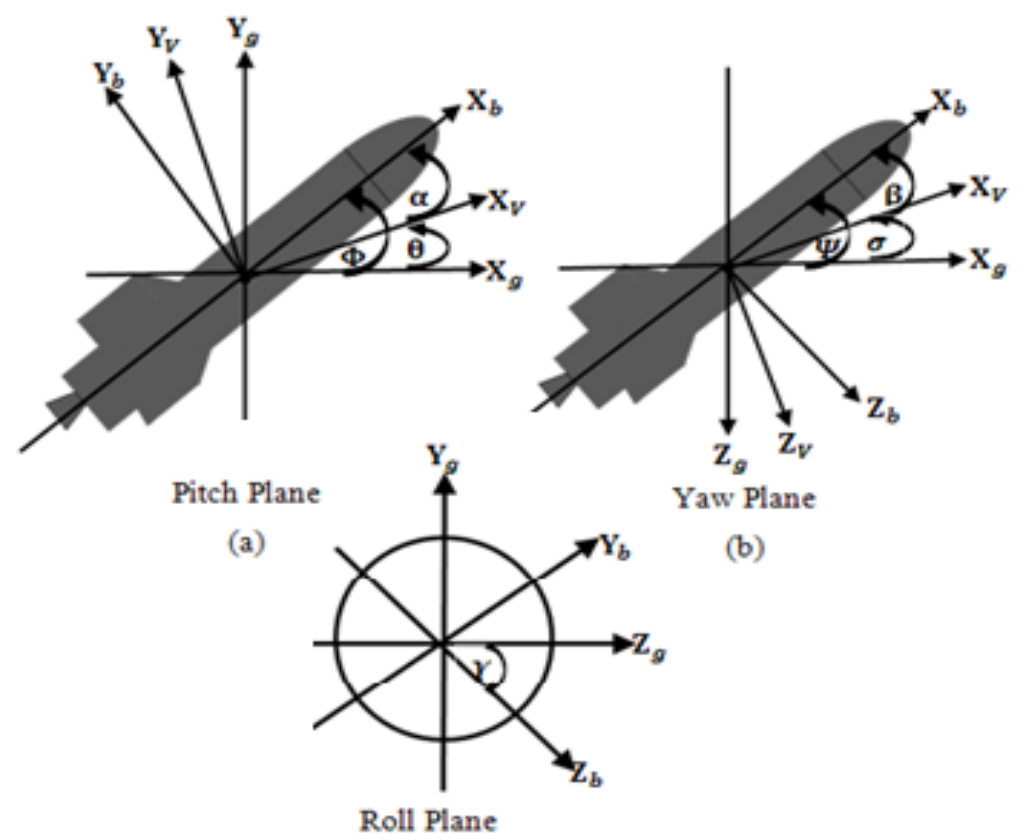

(c)

Fig. 1. The angles between different coordinate systems 
The relationship between the velocity and the body coordinate systems can be obtained as follows

$$
\left[\begin{array}{c}
X_{b} \\
Y_{b} \\
Z_{b}
\end{array}\right]=\left[\begin{array}{ccc}
\cos (\beta) \cos (\alpha) & \cos (\beta) \sin (\alpha) & -\sin (\beta) \\
-\sin (\alpha) & \cos (\alpha) & 0 \\
\sin (\beta) \cos (\alpha) & \sin (\beta) \sin (\alpha) & \cos (\beta)
\end{array}\right]\left[\begin{array}{l}
X_{v} \\
Y_{v} \\
Z_{v}
\end{array}\right]
$$

The velocity and body axes system as well as moments, forces and other quantities are shown in Fig. 2

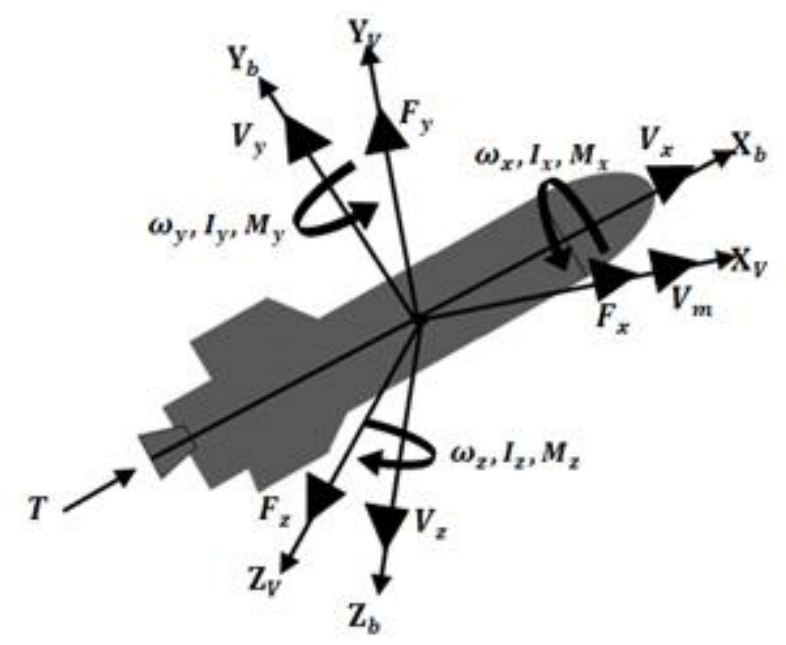

Fig. 2. Moments, forces and other quantities

There are 6 dynamic equations ( 3 for rotational motion and 3 for translation motion) and 6 kinematic equations ( 3 for rotational motion and 3 for translational motion) for a missile with six degrees of freedom. The equations are slightly simpler, if the mass is constant. The missile $6 \mathrm{DOF}$ equations in velocity coordinate system are obtained as following $[1,12,14,15]$.

$$
\begin{aligned}
& F_{x}=m \dot{V}_{m} \\
& F_{y}=m V_{m} \dot{\theta} \\
& F_{z}=-m V_{m} \cos (\theta) \dot{\sigma} \\
& M_{x}=I_{x} \dot{\omega}_{x}-\left(I_{y}-I_{z}\right) \omega_{y} \omega_{z} \\
& M_{y}=I_{y} \dot{\omega}_{y}-\left(I_{z}-I_{x}\right) \omega_{z} \omega_{x} \\
& M_{z}=I_{z} \dot{\omega}_{z}-\left(I_{x}-I_{y}\right) \omega_{x} \omega_{y} \\
& \dot{X}=V_{m} \cos (\theta) \cos (\sigma) \\
& \dot{Y}=V_{m} \sin (\theta) \\
& \dot{Z}=-V_{m} \cos (\theta) \sin (\sigma) \\
& \dot{\Psi}=\left(\omega_{y} \cos (\Upsilon)-\omega_{z} \sin (\Upsilon)\right) / \cos (\Phi) \\
& \dot{\Phi}=\omega_{y} \sin (\Upsilon)+\omega_{z} \cos (\Upsilon) \\
& \dot{Y}=\omega_{x}-\tan (\Phi)\left(\omega_{y} \cos (\Upsilon)-\omega_{z} \sin (\Upsilon)\right) \\
& \dot{\alpha}=\dot{\Phi}-\dot{\theta} \\
& \dot{\beta}=\dot{\Psi}-\dot{\sigma}
\end{aligned}
$$


In these equations, $M_{x}, M_{y}, M_{z}$ are moments acting on missile in body coordinate system; $F_{x}, F_{y}, F_{z}$ are component of forces acting on missile in velocity coordinate system; $I_{x}, I_{y}, I_{z}$ are moments of inertia in body coordinate system; $\omega_{x}, \omega_{y}, \omega_{z}$ are angular velocity in body coordinate system; $X$ is missile range; $Z$ is horizontal displacement of the missile; $Y$ is missile altitude; and $\mathrm{m}$ is missile mass. The moments and the forces acting on missile are due to thrust, aerodynamic and gravity moments and forces are obtained as following $[1,11,12,14-16]$.

$$
\begin{aligned}
& F_{x}=T \cos \left(\alpha-\delta_{\alpha}\right) \cos \left(\beta-\delta_{\beta}\right)-Q S\left(C_{x 0}+C_{x}\left(\alpha^{2}+\beta^{2}\right)\right)-m g \sin (\theta) \\
& F_{y}=T \sin \left(\alpha-\delta_{\alpha}\right)+Q S C_{y} \alpha-m g \cos (\theta) \\
& F_{z}=-T \cos \left(\alpha-\delta_{\alpha}\right) \sin \left(\beta-\delta_{\beta}\right)-Q S C_{z} \beta \\
& M_{x}=D Q S m_{x 0} \frac{\omega_{x} D}{2 V_{m}} \\
& M_{y}=-T \cos \left(\delta_{\alpha}\right) \sin \left(\delta_{\beta}\right) X_{c g}+D Q S\left(m_{y \beta} \beta+m_{y 0} \frac{\omega_{y} D}{V_{m}}\right) \\
& M_{z}=T \sin \left(\delta_{\alpha}\right) X_{c g}+D Q S\left(m_{z \alpha} \alpha+m_{z 0} \frac{\omega_{z} D}{V_{m}}\right)
\end{aligned}
$$

In these equations, $m_{x 0}, m_{y \beta}, m_{y 0}, m_{z \alpha}, m_{z 0}$ are aerodynamic moment coefficients; $C_{x}, C_{x 0}, C_{y}, C_{z}$ are aerodynamic force coefficient; $S$ is the reference area; $D$ is the diameter of maximum cross section area of body; $Q$ is the dynamic pressure; $\delta_{\alpha}$ is the pitch nozzle deflection angle $; \delta_{\beta}$ is the yaw nozzle deflection angle; $T$ is the thrust force; $X_{c g}$ is the distance between the nozzle and center of gravity (c.g); and $g$ is gravity acceleration and is taken to be constant $9.81 \mathrm{~m} / \mathrm{sec}^{2}$.

\section{Gain Schedule Fractional PID Controller Design}

In recent years, researchers reported that controllers utilize fractional order derivatives and integrals to fulfill performance and robustness results those obtained with conventional (integer order) controllers. The Fractional-order PID controller (FPID) controller is the extension of the conventional PID controller depend on fractional calculus. The theories of fractional calculus are explained obviously in, [1].

\subsection{Basic Concepts of FOPID Controller PI $\lambda$ D $\delta$}

The differential equation of the $P I^{\lambda} D^{\delta}$ controller is depicted in time domain by:

$$
u(t)=k_{p} e(t)+k_{i} D_{t}^{-\lambda} e(t)+k_{d} D_{t}^{\delta} e(t)
$$

The continuous transfer function of the $P I^{\lambda} D^{\delta}$ controller is given through Laplace transform

$$
G_{c}(s)=k_{p}+k_{i} s^{-\lambda}+k_{d} s^{\delta}
$$

It is clear that the FOPID controller not only need to design three parameters $k_{p}, k_{i}$ and $k_{d}$, but also need to design two orders $\lambda, \delta$ of integral and derivative controllers. The orders $\lambda, \delta$ are not necessarily integer, but any real numbers [1]. 


\subsection{Optimal Tuning FPID Control Parameters}

Tuning a FPID controller involves setting the proportional, integral, derivative, fractional derivative and fractional integral values to get the best possible control for a particular process. Simulink Design Optimization software uses optimization methods to find parameter values that permit a feasible solution with the given constraints. Simulink Design Optimization software is called the nonlinear control design blockset $(N C D),[17,18]$. This software has characteristics to optimize design criteria in any Simulink model by adjusting selected parameters that have physical limits. Rise time, settling time, overshoot, and saturation limits are design requirements in Simulink response optimization. Each of the step response characteristics is described in the

Fig. 3. A Simulink design optimization procedure is displayed in Fig. 4, [18, 19].

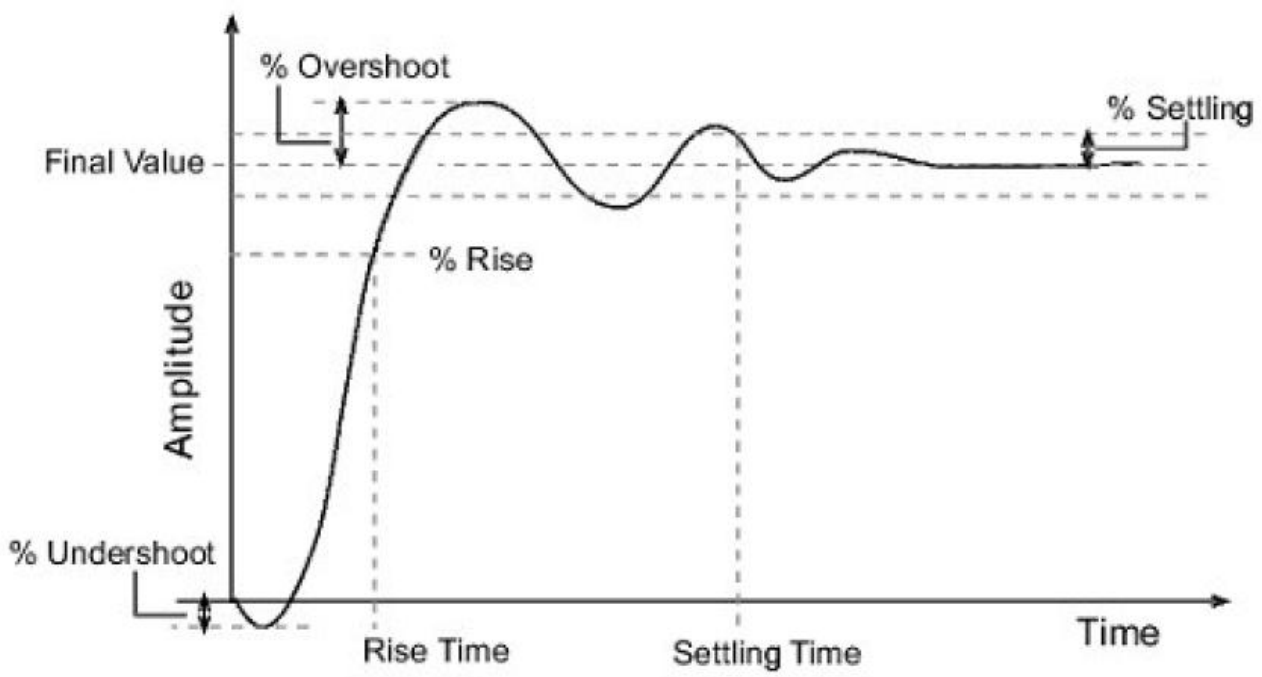

Fig. 3. Step response characteristics

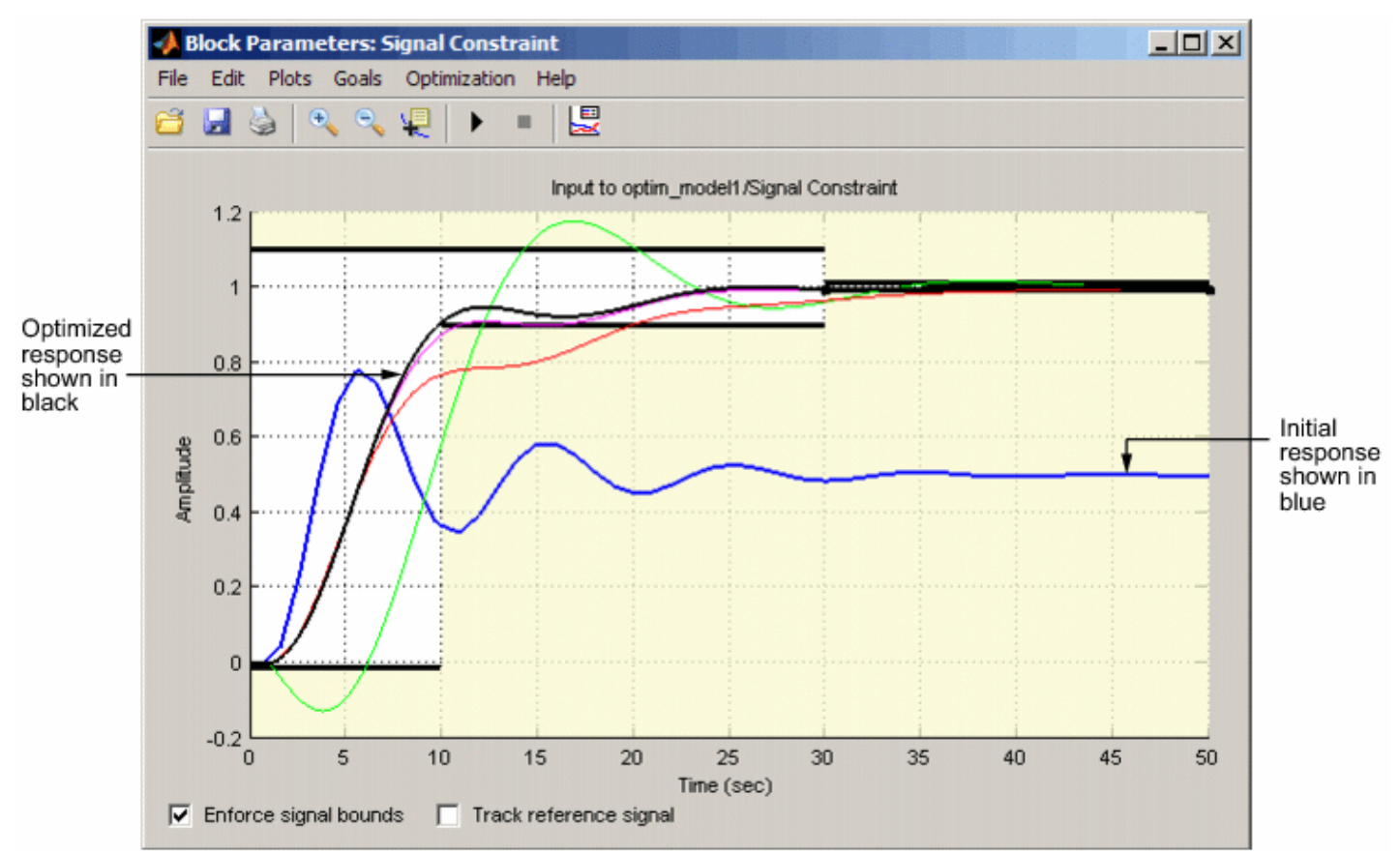

Fig. 4. Simulink design optimization procedures 


\subsection{Gain Scheduling Controller}

In many cases, the dynamics of a process are changed with the operating conditions of the process. It is possible to change the parameters of the controller by observing the operating conditions of the process. This method is called gain scheduling, since the scheme was used to accommodate alteration in process gain. Gain scheduling is simple to achieve in computercontrolled systems, supplied that there is support in the available software. Gain scheduling depend on measurements of procedures of the process is the best way to compensate for changes in process parameters or known nonlinearities. If we utilize the informal definition of adaptive controller, Gain scheduling is a very useful method for decreasing the effects of parameter variations. There are also several commercial process control systems where gain scheduling can be used to compensate for static and dynamic nonlinearities. Split-range controllers that utilize different sets of parameters for different ranges of the process output can be considered as a special kind of gain-scheduling controllers. It is possible to find auxiliary variables that correlate well with the changes in process dynamics. It is possible to decrease the effects of parameter variations by changing the parameters of the controller as functions of the auxiliary variables. Gain scheduling can be seen as a feedback control system where the feedback gains are tuned by utilizing feed forward compensation. The essential problem in the design of systems with gain scheduling is to get appropriate scheduling variables. This is done on the basis of information of the physics of the systems [20-22]. The general block diagram of gain schedule controller is shown in Fig. 5

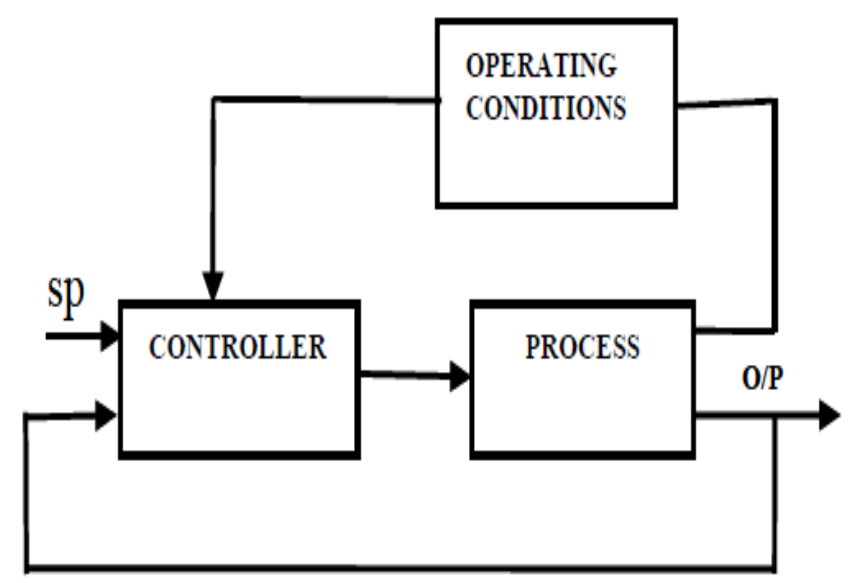

Fig. 5. General block diagram of Gain scheduled Controller

When scheduling variables are founded, the controller parameters are calculated at a number of operating conditions by utilizing some appropriate design method. The controller is tuned for each operating condition. The stability and performance of the system are evaluated by simulation.

\section{Control Application and Results}

In this section, the autonomous flight of six degree of freedom flying body is simulated. The goal is to control the trajectory of the flight path of six degree of freedom flying body model using gain schedule fractional PID controller. The design of gain schedule fractional PID controller for six degree of freedom flying body is described. This design has been implemented in a simulation environment under Matlab's toolbox Simulink and results will be given and compared,[1, 10, 15, 23]. 


\subsection{Model Description}

Missile solid propellant thrust will be divided into two main phases (1) Boost phase: that will start at beginning of flight until the time $5.8 \mathrm{sec}(0 \leq \mathrm{t}<5.8 \mathrm{sec})$ and thrust force is maximum $\left(T_{\max }\right)$. (2)Sustain phase: that will start after boost phase until the impact with target $(5.8 \leq \mathrm{t}<$ $25 \mathrm{sec}$ ) and thrust force is minimum $\left(T_{\min }\right)$. The thrust force curve is represented in Fig. 6.

The pitch nozzle deflection angle $\left(\delta_{\alpha}\right)$ and yaw nozzle deflection plane $\left(\delta_{\beta}\right)$ are limited with $\pm 22.9^{\circ}( \pm 0.4 \mathrm{rad})$.

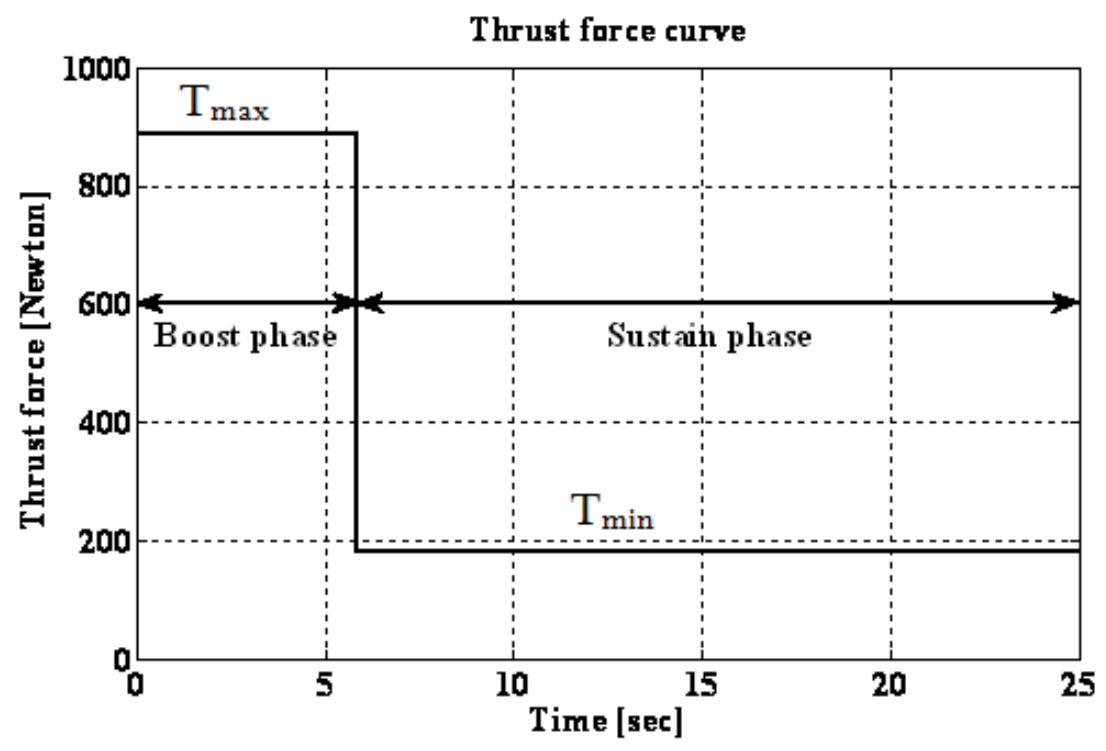

Fig. 6. Thrust force curve

\subsection{Controller Design}

In this section FPID controller in pitch and yaw channels are designed and then gain schedule FPID in pitch channel only is designed and the results are compared. The fractional PID controller has five unknown parameter $k_{p}, k_{i}, k_{d}, \lambda$ and $\delta$ are designed and tuned by Simulink design optmization through signal constraint that was explained previously.

The optimized parameter of fractional PID controller as following: The fractional PID controller gains for pitch angle are shown in Table 1

Table 1. The optimized parameters of FPID controller for pitch channel

\begin{tabular}{|c|c|c|c|c|}
\hline $\boldsymbol{k}_{\boldsymbol{p}}$ & $\boldsymbol{k}_{\boldsymbol{i}}$ & $\boldsymbol{\lambda}$ & $\boldsymbol{k}_{\boldsymbol{d}}$ & $\boldsymbol{\delta}$ \\
\hline 18.47 & 29.9 & 0.943 & 4.709 & 0.6608 \\
\hline
\end{tabular}

The fractional PID controller gains for yaw angle are shown in Table 2

Table 2. The optimized parameters of FPID controller for yaw channel

\begin{tabular}{|c|c|c|c|c|}
\hline $\boldsymbol{k}_{\boldsymbol{p}}$ & $\boldsymbol{k}_{\boldsymbol{i}}$ & $\boldsymbol{\lambda}$ & $\boldsymbol{k}_{\boldsymbol{d}}$ & $\boldsymbol{\delta}$ \\
\hline-18.47 & -29.9 & 0.943 & -4.709 & 0.6608 \\
\hline
\end{tabular}

The optimized parameters of gain schedule FPID controller for pitch channel at boost phase $(0 \leq t<5.8 \mathrm{sec})$ are seen in Table 3 
Table 3. The optimized parameters of gain schedule FPID controller for pitch channel at boost phase

\begin{tabular}{|c|c|c|c|c|}
\hline $\boldsymbol{k}_{\boldsymbol{p}}$ & $\boldsymbol{k}_{\boldsymbol{i}}$ & $\boldsymbol{\lambda}$ & $\boldsymbol{k}_{\boldsymbol{d}}$ & $\boldsymbol{\delta}$ \\
\hline 21.934 & 91.4234 & 0.9241 & 0.3592 & 0.949 \\
\hline
\end{tabular}

The optimized parameters of gain schedule FPID controller for pitch channel at sustain phase $(5.8 \leq t<25 \mathrm{sec})$ are seen in Table 4

Table 4. The optimized parameters of gain schedule FPID controller for pitch channel at sustain phase

\begin{tabular}{|c|c|c|c|c|}
\hline $\boldsymbol{k}_{\boldsymbol{p}}$ & $\boldsymbol{k}_{\boldsymbol{i}}$ & $\boldsymbol{\lambda}$ & $\boldsymbol{k}_{\boldsymbol{d}}$ & $\boldsymbol{\delta}$ \\
\hline 155.0734 & 261.7472 & 1.0808 & 298.509 & 0.244 \\
\hline
\end{tabular}

\subsection{Simulation Results}

Fig.7 represents the pitch error, the difference between pitch angle and pitch demand, comparison between fractional PID controller and gain schedule fractional PID controller in boost phase. The steady state error for gain schedule fractional PID controller is less than the steady state error for fractional PID controller.

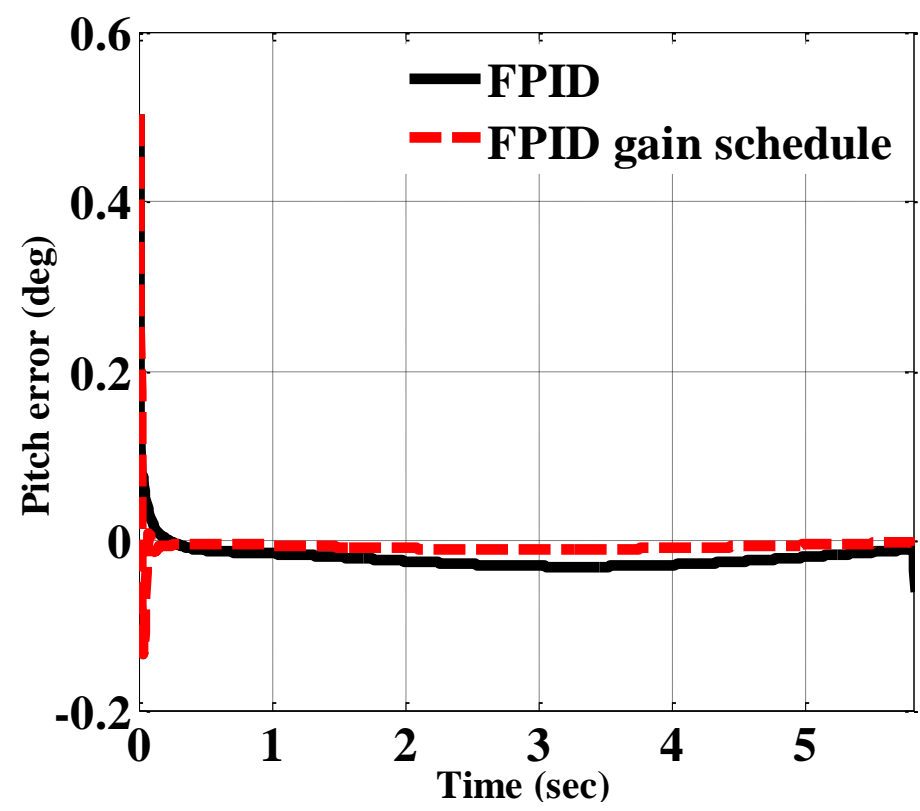

Fig.7. Pitch error comparison between FPID and gain schedule FPID in boost phase

Fig. 8 represents the pitch error comparison between fractional PID controller and gain schedule fractional PID controller in sustain phase. The error for fractional PID controller has high overshoot at time $5.8 \mathrm{sec}$ but the error for gain schedule fractional PID controller does not have any overshoot. 
Fig. 9 depicts the pitch actuator action comparison between fractional PID controller and gain schedule fractional PID controller.

Fig. 10 displays the pitch actuator action at starting of boost phase comparison between fractional PID controller and gain schedule fractional PID controller. The down overshoot in gain schedule fractional PID controller is less than that of fractional PID controller

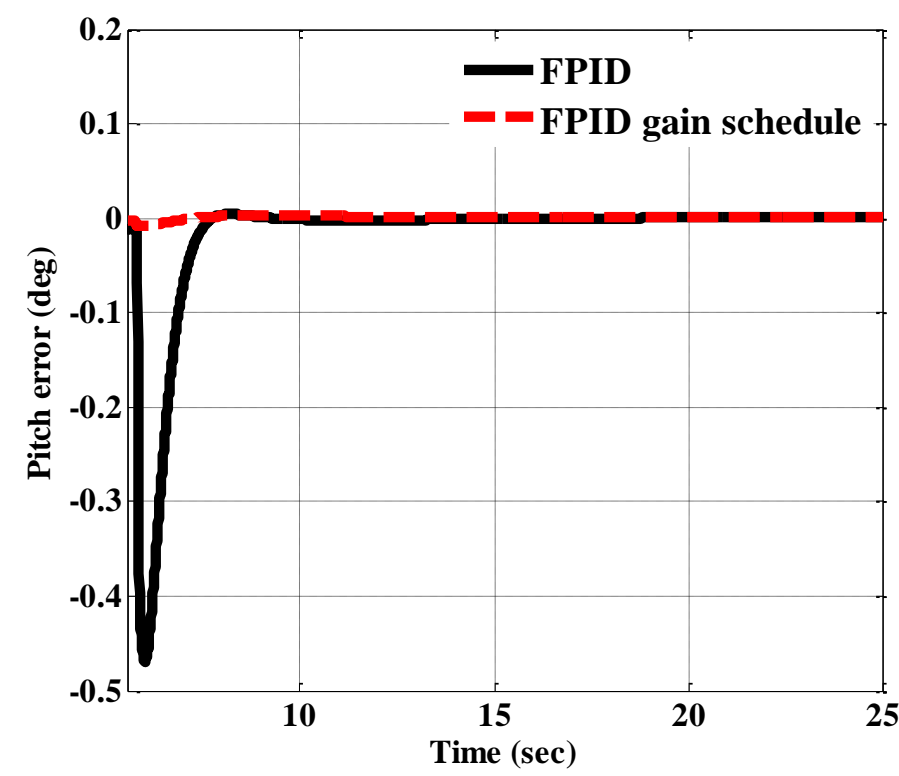

Fig. 8. Pitch error comparison between FPID and gain schedule FPID in sustain phase

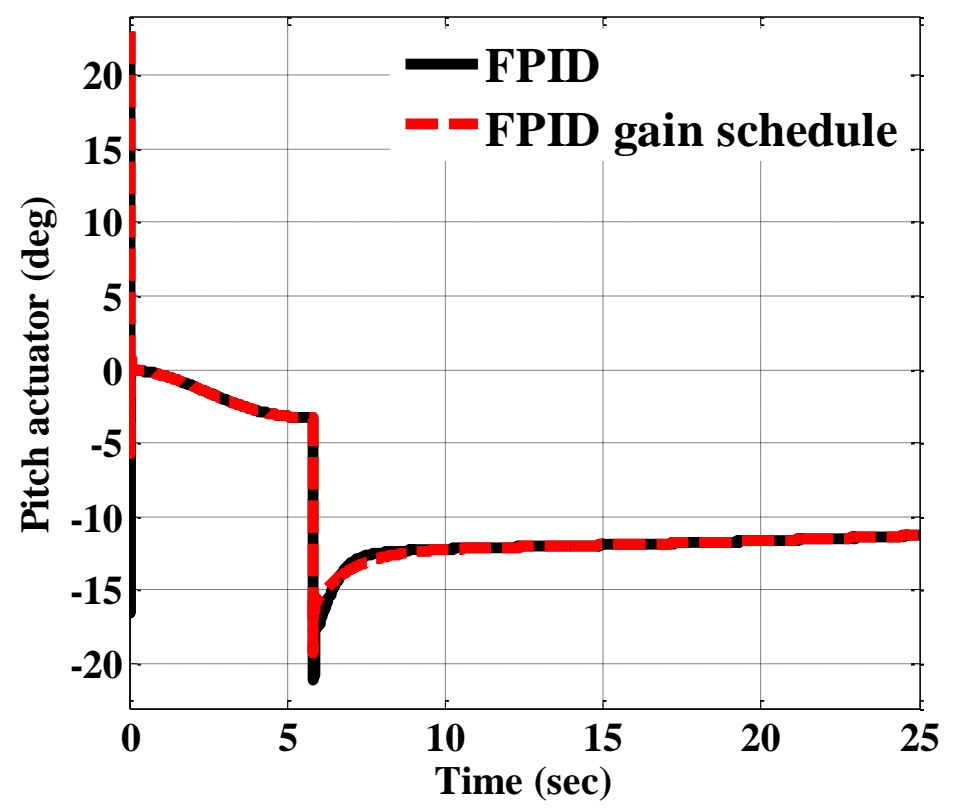

Fig. 9 . Pitch actuator comparison between FPID and gain schedule FPID 


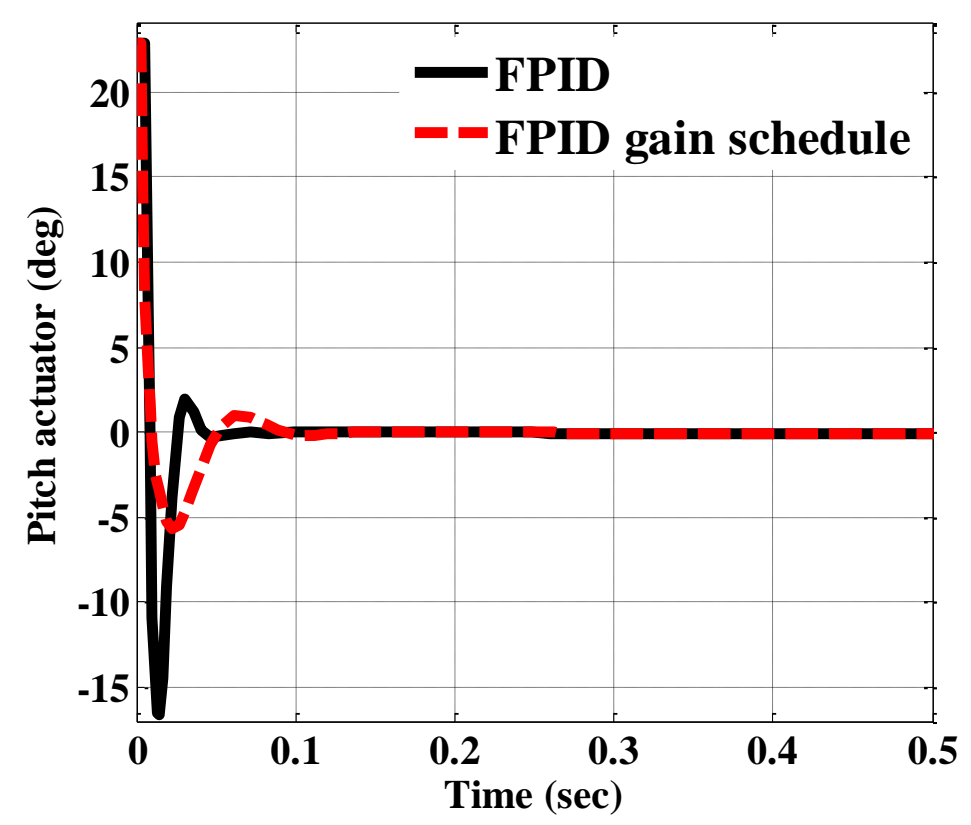

Fig. 10. pitch actuator at starting boost phase comparison between FPID and gain schedule FPID

\subsection{Wind Effect}

The wind effect is studied where wind velocity magnitude in

Fig. 11 is summated to missile velocity $\left(V_{m}\right)$ in the same direction in and results is compared . Fig. 12 depicts the wind effect on the pitch error where the wind in gain schedule fractional PID controller is slightly small but in fractional PID controller the wind effect is increased.

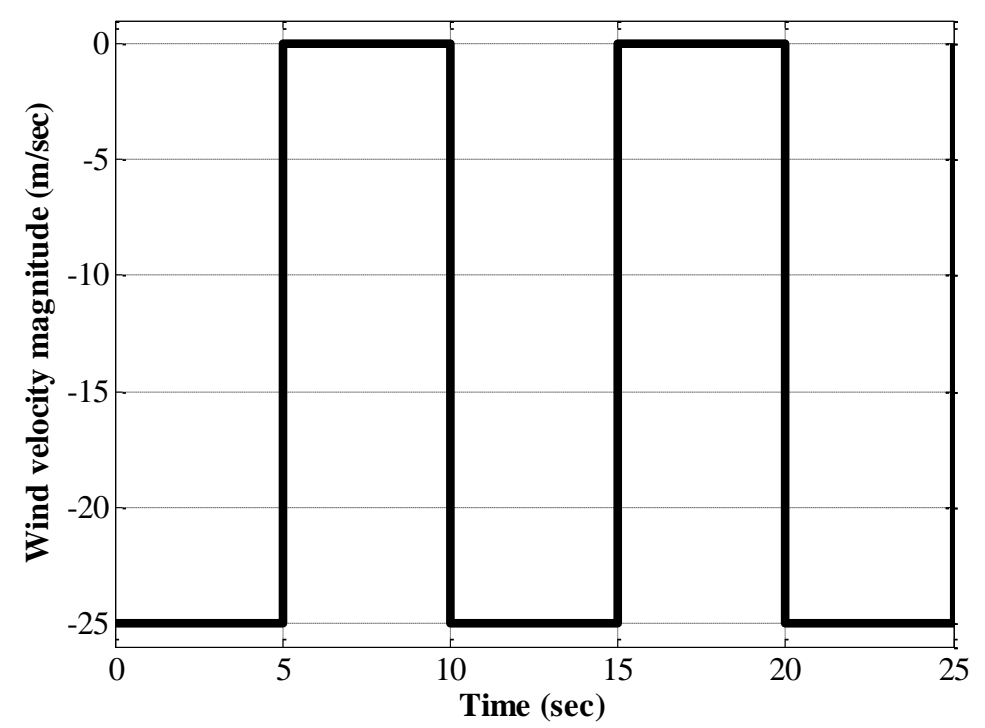

Fig. 11. Wind velocity magnitude 


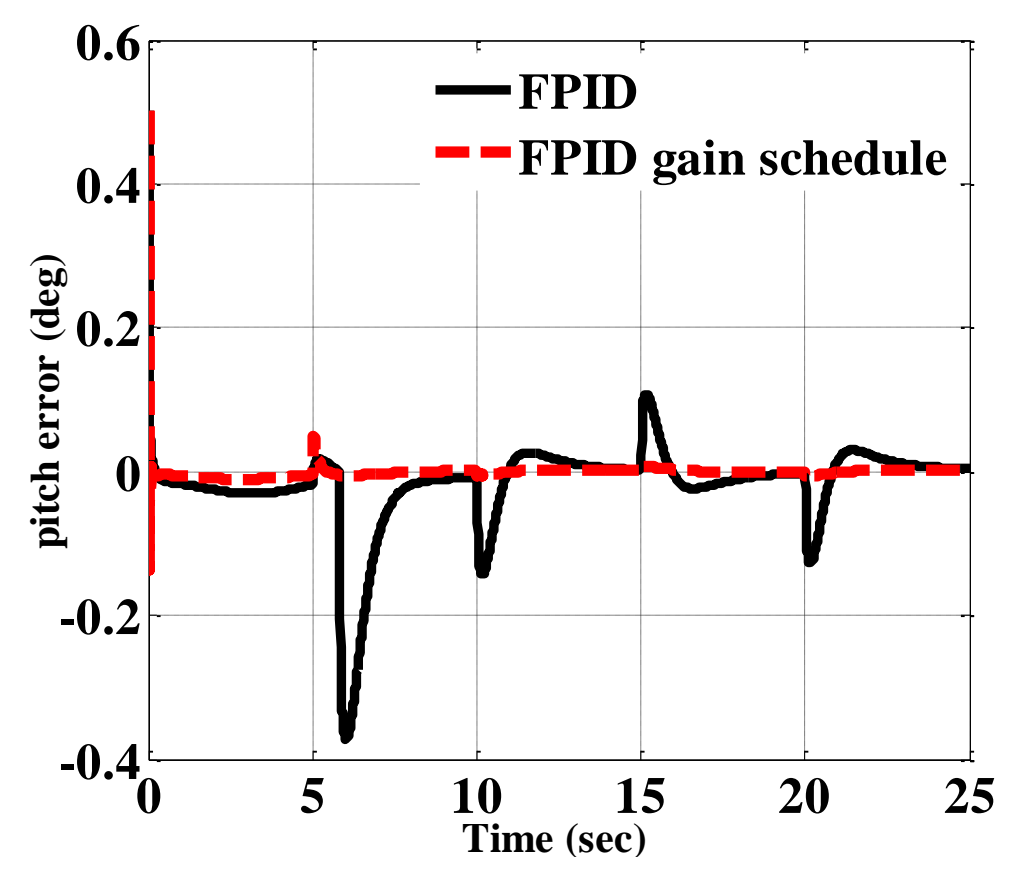

Fig. 12. Pitch error comparison between FPID and gain schedule

FPID with wind effect

Fig. 13 displays the pitch actuator action due to the wind effect for FPID and gain schedule FPID controller.

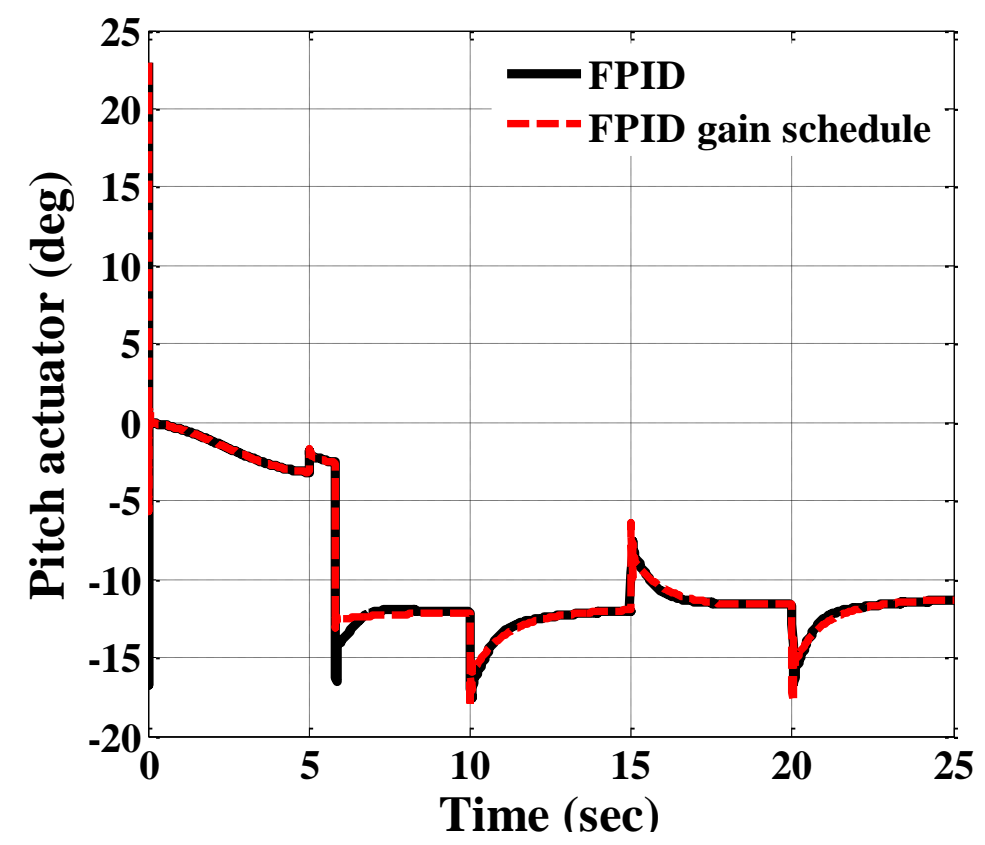

Fig. 13. Pitch actuator comparison between FPID and gain schedule FPID with wind effect 
Fig. 14 displays the yaw actuator action due to the wind effect for FPID and gain schedule FPID controller. The yaw actuator is chattered in the boost phase due to the wind effect

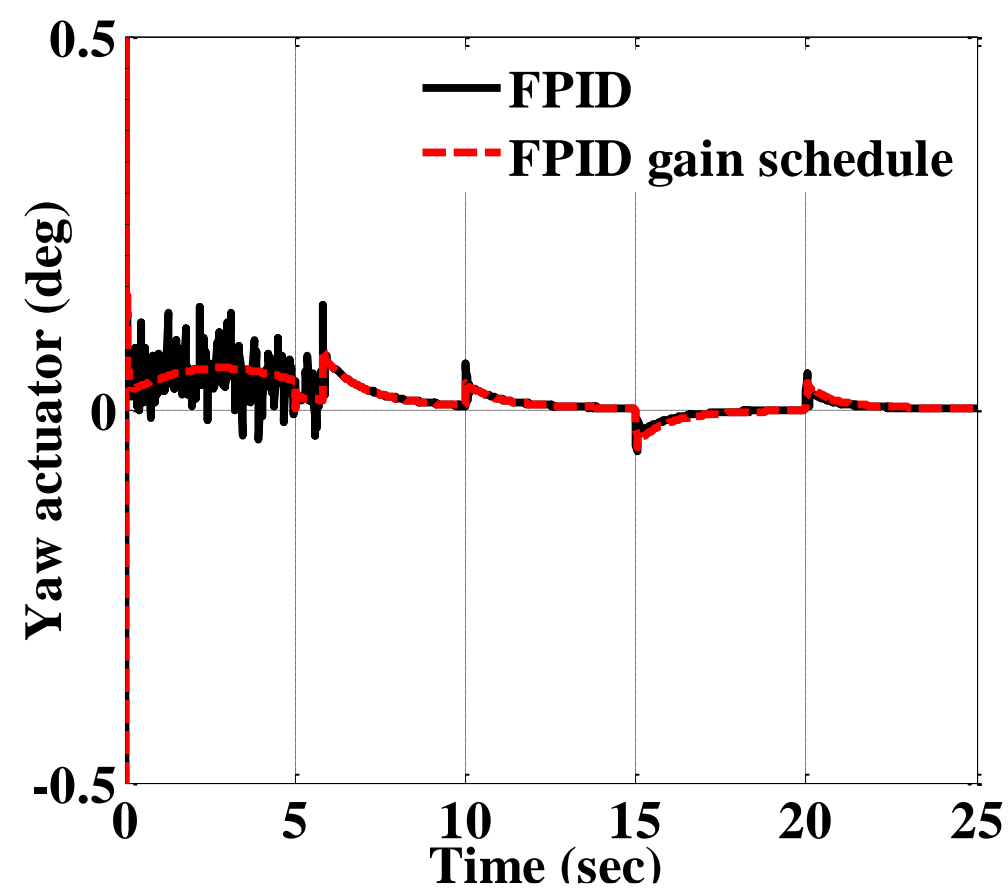

Fig. 14. Yaw actuator comparison between FPID and gain schedule FPID with wind effect

\section{Conclusion}

The design of gain schedule fractional PID controller gives more accurate tracking with demand program where there is no steady state error. The design of gain schedule fractional PID controller gave the best response for pitch and yaw angles since there are no oscillation (chattering) in yaw actuator, and there is no error overshoot at $t=5.8 \mathrm{sec}$. The response with gain schedule FPID is slightly changed due to wind effect.

\section{References}

[1] M. A. Aboelela, M. F. Ahmed, and H. T. Dorrah, "Design of aerospace control systems using fractional PID controller," Journal of Advanced Research, vol. 3, pp. 225-232, 2012.

[2] M. Fawzy, M. Aboelela, O. A. El Rhman, and H. Dorrah, "Design of Intelligent Missile Control System Using Model Predictive Control," 2010.

[3] D. A. MacKenzie, Inventing accuracy: A historical sociology of nuclear missile guidance: MIT press, 1993.

[4] C. Draper, "Guidance is forever," Navigation, vol. 18, pp. 26-50, 1971.

[5] M. L. Spearman, Historical development of worldwide guided missiles: NASA Langley Research Center, 1983.

[6] R. H. Battin, "Space guidance evolution-A personal narrative," Journal of Guidance, Control, and Dynamics, vol. 5, pp. 97-110, 1982. 
[7] M. W. Fossier, "The development of radar homing missiles," Journal of Guidance, Control, and Dynamics, vol. 7, pp. 641-651, 1984.

[8] W. Haeussermann, "Developments in the field of automatic guidance and control of rockets," Journal of Guidance, Control, and Dynamics, vol. 4, pp. 225-239, 1981.

[9] A. S. Locke, Guidance: Principles of Guided Missile Design: Van Nostrand, 1955.

[10] R. Westrum, Sidewinder: creative missile development at China Lake: Naval Institute Press, 2013.

[11] J. H. Blakelock, Automatic control of aircraft and missiles: John Wiley \& Sons, 1991.

[12] M. Fawzy, M. Aboelela, O. A. El Rhman, and H. Dorrah, "Design of Missile Control System Using Model Predictive Control," Online Journal on Computer Science and Information Technology (OJCSIT), vol. 1, 2011.

[13] G. M. Siouris, Missile guidance and control systems: Books24x7. com, 2005.

[14] T. Ashish, "Modern control design with Matlab and simulink," Indian Institute of Technology, Kanpur, India, John Wiley \& Sons, 2002.

[15] G. M. Siouris, Missile guidance and control systems: Springer Science \& Business Media, 2004.

[16] P. Garnell, Z. Qi, and Q. Xia, Guided weapon control systems: Beijing institute of technology, 2003.

[17] A. K. Tyagi, MATLAB and SIMULINK for Engineers: Oxford University Press, 2012.

[18] I. MathWorks, MATLAB: the language of technical computing. Desktop tools and development environment, version 7.10.0 vol. 9: MathWorks, 2010.

[19] M. A. Wael and Q. Quan, "Robust hybrid control for ballistic missile longitudinal autopilot," Chinese Journal of Aeronautics, vol. 24, pp. 777-788, 2011.

[20] S. S. Pradeepkannan D "Implementation of Gain Scheduled PID Controller for a Nonlinear Coupled Spherical Tank Process," International Journal of Mechanical \& Mechatronics Engineering IJMME, vol. 14, p. 6, December 2014

[21] M. KRISHNA, S. RAO, and R. RAJU, "Theoretical and experimental investigation of gain scheduling and adaptive autopilots for a model boat," International Journal of Engineering Science and Technology, vol. 3, pp. 902-911, 2011.

[22] D. O. Araromi and A. A. Sulayman, "GAIN SCHEDULING CONTROL DESIGN FOR SHELL HEAVY OIL FRACTIONATOR COLUMN."

[23] I. MathWorks, MATLAB: the language of technical computing. Desktop tools and development environment, version 7 vol. 9: MathWorks, 2005. 